\title{
Exact solution for the flow of Oldroyd-B fluid due to constant shear and time dependent velocity
}

\author{
Vatsala Mathur ${ }^{1}$, Kavita Khandelwal ${ }^{1}$ \\ ${ }^{I}$ (Department of Mathematics, Malaviya National Institute of Technology, Jaipur, India)
}

\begin{abstract}
In this paper, we use the finite Hankel and Laplace transforms to determine the velocity field corresponding to the flow of Oldroyd-B fluid in the annular region between two infinitely long coaxial cylinders. Initially, the fluid is at rest and the motion is produced by the inner cylinder pulled with a constant shear and outer cylinder moving with time dependent velocity. The obtained solution is presented under a series form in terms of the generalized G functions. Finally, the influence of different values of parameters, constants and fractional coefficient on the velocity field are also analyzed using graphical illustration.
\end{abstract}

Keywords: Fractional Calculus, Hankel transform, Laplace transform, Oldroyd-B fluid, Velocity field.

\section{Introduction}

From industries and engineering point of view, various fluids with complicated rheological properties cannot be specified as Newtonian fluids. These fluids are called Non-Newtonian fluids. Few examples of such fluids are slurries, lava, blood, polymers etc. The flow behavior of such fluids cannot be described by classical Navier-Stokes theory due to their non-linear viscoelastic behavior. Hence, many models have been proposed for non-Newtonian fluids such as differential type, rate type and integral type etc. Among all of these, rate type model has got much attention.

Ting [1] has given first exact solution corresponding to motion of second grade fluids in cylindrical domains. The objective of his work was to apply Coleman and Noll's theory to investigate certain non-steady flows of second-order fluids. The first exact solution corresponding to motion of Maxwell fluids in cylindrical domain has been determined by Srivastava [2] .Waters and King [3] published first exact solution corresponding to motion of Oldroyd-B fluids in cylindrical domains. The first exact solution for the motion of second grade fluids due to a shear stress on the boundary has been determined by Bandelli and Rajagopal [4].He studied a number of unidirectional transient flows of a second grade fluid in a domain with one finite dimension. Recently, many papers regarding such motions have been published [5-12]. Fetecau [13] used constitutive relation as follows

$$
\left(1+\lambda \partial_{t}\right) \tau=\mu\left(1+\lambda_{r} \partial_{t}\right) \partial_{r} v(r, t),
$$

where $\lambda$ and $\lambda_{r}$ are relaxation and retardation times, $\tau$ is tangential tension, $\mu$ is the dynamic viscosity and $v$ is the velocity.

Using fractional approach, the constitutive relation of the generalized Oldroyd-B fluid is written as

$$
\left(1+\lambda D_{t}^{\alpha}\right) \tau=\mu\left(1+\lambda_{r} D_{t}^{\beta}\right) \partial_{r} v(r, t),
$$

where $D_{t}^{\alpha}$ and $D_{t}^{\beta}$ are fractional operators and are defined as [14]

$$
D_{t}^{\alpha} f(t)=\left\{\begin{array}{lr}
\frac{1}{\Gamma(1-\alpha)} \frac{d}{d t} \int_{0}^{t} \frac{f(\tau)}{(t-\tau)^{\alpha}} d \tau, & 0 \leq \alpha<1 ; \\
\frac{d}{d t} f(t), & \alpha=1,
\end{array}\right\}
$$

where $\Gamma($.$) is the Gamma function.$

The aim of this paper is to provide exact solution for the velocity field of flow for Oldroyd-B fluid between two infinitely long coaxial cylinders, where inner cylinder is pulled with constant shear and outer cylinder is moving with time dependent velocity. This solution is obtained by using finite Hankel and Laplace transform methods and the result is presented in terms of the generalized-G functions.

\section{Governing equations}

Let us consider the unsteady flow of an incompressible Oldroyd-B fluid in coaxial cylinders. The following assumptions are considered during this mathematical study. The flows are assumed to be axi- 
symmmetric. The fluid velocity at the direction of the pipe radius is assumed to be zero. The axial velocity is assumed to be only relevant to the cylinder radius.

The equation of axial flow motion is written as [5]

$$
\rho \frac{\partial v}{\partial t}=\frac{\partial \tau}{\partial r}+\frac{1}{r} \tau-\frac{\partial p}{\partial z}
$$

where $\rho$ is the constant density of the fluid.

Substitute Eq. (2) into Eq. (4), we get

$\left(1+\lambda D_{t}^{\alpha}\right) \frac{\partial v}{\partial t}=A+\lambda A \frac{t^{-\alpha}}{\Gamma(1-\alpha)}+v\left(1+\lambda_{r} D_{t}^{\beta}\right)\left(\partial_{r}^{2}+\frac{1}{r} \partial_{r}\right) v(r, t)$,

where $v=\frac{\mu}{\rho}$ is the kinematical viscosity and $-A \rho=\frac{\partial p}{\partial z}$ is the constant pressure gradient that acts on the liquid in the z-direction.

\section{Flow through the annular region}

Consider an Oldroyd-B fluid at rest between two infinitely long coaxial cylinders. Also, consider that radius of inner and outer cylinders are $R_{1}$ and $R_{2}\left(>R_{1}\right)$ respectively. The inner cylinder pulled with constant shear and outer cylinder is moving with time dependent velocity. We have to solve the next initial and boundary problem, in the absence of a pressure gradient in the z-direction.

$\left(1+\lambda D_{t}^{\alpha}\right) \frac{\partial v}{\partial t}=v\left(1+\lambda_{r} D_{t}^{\beta}\right)\left(\partial_{r}^{2}+\frac{1}{r} \partial_{r}\right) v(r, t), \quad \mathrm{R}_{1}<r<\mathrm{R}_{2}, t>0$.

The initial and boundary conditions are expressed by

$v(r, 0)=0, \quad \partial_{\mathrm{t}} v(r, 0)=0, \quad \mathrm{R}_{1} \leq r \leq \mathrm{R}_{2}$,

$\left.\mu\left(1+\lambda_{r} D_{t}^{\beta}\right) \partial_{r} v(r, t)\right|_{r=R_{1}}=f_{1}, \quad v\left(R_{2}, t\right)=f_{2} t^{p}, \quad \mathrm{t}>0, \quad \mathrm{p} \geq 0$,

where $f_{1}, f_{2}$ are constant.

Making the change to unknown function

$v(r, t)=V(r)+u(r, t)$,

where

$V(r)=\frac{R_{1} f_{1}}{\mu} \ln \left(r / R_{2}\right)$.

Substitute Eq. (9) into Eq. (6), we get

$\left(1+\lambda D_{t}^{\alpha}\right) \frac{\partial u(r, t)}{\partial t}=v\left(1+\lambda_{r} D_{t}^{\beta}\right)\left(\partial_{r}^{2}+\frac{1}{r} \partial_{r}\right) u(r, t)$.

Substitute Eq. (9) into Eqs. (7) and (8), we get

$u(r, 0)=-V(r), \quad \partial_{\mathrm{t}} u(r, 0)=0$,

$\mu\left(1+\lambda_{r} D_{t}^{\beta}\right) \partial_{r} u\left(R_{1}, t\right)=-f_{1} \lambda_{r} \frac{t^{-\beta}}{\Gamma(1-\beta)}, \quad u\left(R_{2}, t\right)=f_{2} t^{p}, \quad \mathrm{t}>0, \quad \mathrm{p} \geq 0$.

The Hankel Transform method with respect to $r$ is used and is defined as follows [14]

$\bar{u}=\int_{R_{1}}^{R_{2}} r u(r, s) \phi_{1}\left(s_{n}, r\right) d r$.

The inverse Hankel Transform as defined by [14]

$u(r, s)=\frac{\pi^{2}}{2} \sum_{n=1}^{\infty} \frac{s_{n}^{2} J_{0}^{2}\left(R_{2} s_{n}\right) \bar{u}\left(s_{n}, s\right) \phi_{1}\left(s_{n}, r\right)}{J_{1}^{2}\left(R_{1} s_{n}\right)-J_{0}^{2}\left(R_{2} s_{n}\right)}$,

where $\phi_{1}\left(s_{n}, r\right)=J_{1}\left(R_{1} s_{n}\right) Y_{0}\left(s_{n} r\right)-Y_{1}\left(R_{1} s_{n}\right) J_{0}\left(s_{n} r\right), \mathrm{s}_{\mathrm{n}}$ is the positive root of $\phi_{1}\left(s_{n}, R_{2}\right)=0$.

Applying the Hankel transform to Eq. (11), we obtain 


$$
\begin{aligned}
\left(1+\lambda D_{t}^{\alpha}\right) \frac{\partial \bar{u}\left(s_{n}, t\right)}{\partial t} & =-v s_{n}^{2}\left(1+\lambda_{r} D_{t}^{\beta}\right) \bar{u}\left(s_{n}, t\right)+\frac{2 v f_{1} \lambda_{r}}{\pi s_{n} \mu} \frac{t^{-\beta}}{\Gamma(1-\beta)}-\frac{2 v f_{2} t^{p}}{\pi} \frac{J_{1}\left(R_{1} s_{n}\right)}{J_{0}\left(R_{2} s_{n}\right)} \\
& -\frac{2 v f_{2} \lambda_{r}}{\pi} \frac{J_{1}\left(R_{1} s_{n}\right)}{J_{0}\left(R_{2} s_{n}\right)} \frac{\Gamma(p+1)}{\Gamma(p-\beta+1)} t^{p-\beta} .
\end{aligned}
$$

Applying the Hankel transform to Eq. (12), we obtain

$$
\bar{u}\left(s_{n}, 0\right)=\frac{2 f_{1}}{\pi \mu s_{n}^{3}}, \quad \partial_{\mathrm{t}} \bar{u}\left(s_{n}, 0\right)=0 .
$$

Applying Laplace transform to Eq. (16), we obtain

$$
\begin{aligned}
\bar{u}\left(s_{n}, s\right) & =\bar{u}\left(s_{n}, 0\right) \frac{\left(1+\lambda s^{\alpha}+v s_{n}^{2} \lambda_{r} s^{\beta-1}\right)}{\left(s+\lambda s^{\alpha+1}+v s_{n}^{2}+v s_{n}^{2} \lambda_{r} s^{\beta}\right)} \\
& +\frac{2 v \lambda_{r} f_{1}}{\pi \mu s_{n}} \frac{1}{s^{1-\beta}\left(s+\lambda s^{\alpha+1}+v s_{n}^{2}+v s_{n}^{2} \lambda_{r} s^{\beta}\right)} \\
& -\frac{2 v f_{2} \Gamma(p+1)}{\pi} \frac{J_{1}\left(R_{1} s_{n}\right)}{J_{0}\left(R_{2} s_{n}\right)} \frac{1}{s^{p+1}\left(s+\lambda s^{\alpha+1}+v s_{n}^{2}+v s_{n}^{2} \lambda_{r} s^{\beta}\right)} \\
& -\frac{2 v \lambda_{r} f_{2} \Gamma(p+1)}{\pi} \frac{J_{1}\left(R_{1} s_{n}\right)}{J_{0}\left(R_{2} s_{n}\right)} \frac{1}{s^{p-\beta+1}\left(s+\lambda s^{\alpha+1}+v s_{n}^{2}+v s_{n}^{2} \lambda_{r} s^{\beta}\right)} .
\end{aligned}
$$

Substitute Eq. (17) into Eq. (18), we obtain

$$
\begin{aligned}
\bar{u}\left(s_{n}, s\right) & =\frac{2 f_{1}}{\pi \mu s_{n}} \frac{\left(1+\lambda s^{\alpha}+v s_{n}^{2} \lambda_{r} s^{\beta-1}\right)}{s_{n}^{2}\left(s+\lambda s^{\alpha+1}+v s_{n}^{2}+v s_{n}^{2} \lambda_{r} s^{\beta}\right)} \\
& +\frac{2 v \lambda_{r} f_{1}}{\pi \mu s_{n}} \frac{1}{s^{1-\beta}\left(s+\lambda s^{\alpha+1}+v s_{n}^{2}+v s_{n}^{2} \lambda_{r} s^{\beta}\right)} \\
& -\frac{2 v f_{2} \Gamma(p+1)}{\pi} \frac{J_{1}\left(R_{1} s_{n}\right)}{J_{0}\left(R_{2} s_{n}\right)} \frac{1}{s^{p+1}\left(s+\lambda s^{\alpha+1}+v s_{n}^{2}+v s_{n}^{2} \lambda_{r} s^{\beta}\right)} \\
& -\frac{2 v \lambda_{r} f_{2} \Gamma(p+1)}{\pi} \frac{J_{1}\left(R_{1} s_{n}\right)}{J_{0}\left(R_{2} s_{n}\right)} \frac{1}{s^{p-\beta+1}\left(s+\lambda s^{\alpha+1}+v s_{n}^{2}+v s_{n}^{2} \lambda_{r} s^{\beta}\right)} .
\end{aligned}
$$

Applying Inverse-Laplace transform to Eq. (19) and taking into account the following result [15]

$$
\begin{aligned}
& G_{a, b, c}(d, t)=L^{-1}\left\{\frac{q^{b}}{\left(q^{a}-d\right)^{c}}\right\} \\
&=\sum_{\mathrm{j}=0}^{\infty} \frac{\mathrm{d}^{\mathrm{j}} \Gamma(c+j)}{\Gamma(c) \Gamma(j+1)} \frac{t^{(c+j) a-b-1}}{\Gamma[(c+j) a-b]} ; \\
& \operatorname{Re}(a c-b)>0,\left|\frac{\mathrm{d}}{\mathrm{q}^{\mathrm{a}}}\right|<1,
\end{aligned}
$$

we obtain 


$$
\begin{aligned}
\bar{u}\left(s_{n}, t\right)= & \frac{2 f_{1}}{\pi \mu s_{n}}\left\{\frac{1}{s_{n}^{2}}-\frac{1}{s_{n}^{2}} \sum_{m=0}^{\infty}(-1)^{m}\left(\frac{v s_{n}^{2}}{\lambda}\right)^{m+1} \sum_{k=0}^{m}\left(\begin{array}{l}
m \\
k
\end{array}\right) \lambda_{r}^{k} G_{\alpha, \beta k-m-2, m+1}\left(-\lambda^{-1}, t\right)\right\} \\
& +\frac{2 \lambda_{r} f_{1}}{\pi \mu s_{n}} \frac{1}{s_{n}^{2}} \sum_{m=0}^{\infty}(-1)^{m}\left(\frac{v s_{n}^{2}}{\lambda}\right)^{m+1} \sum_{k=0}^{m}\left(\begin{array}{l}
m \\
k
\end{array}\right) \lambda_{r}^{k} G_{\alpha, \beta k-m+\beta-2, m+1}\left(-\lambda^{-1}, t\right) \\
& -\frac{2 f_{2} \Gamma(p+1)}{\pi} \frac{J_{1}\left(R_{1} s_{n}\right)}{J_{0}\left(R_{2} s_{n}\right)} \frac{1}{s_{n}^{2}} \sum_{m=0}^{\infty}(-1)^{m}\left(\frac{v s_{n}^{2}}{\lambda}\right)^{m+1} \sum_{k=0}^{m}\left(\begin{array}{l}
m \\
k
\end{array}\right) \lambda_{r}^{k} G_{\alpha, \beta k-m-p-2, m+1}\left(-\lambda^{-1}, t\right) \\
& -\frac{2 \lambda_{r} f_{2} \Gamma(p+1)}{\pi} \frac{J_{1}\left(R_{1} s_{n}\right)}{J_{0}\left(R_{2} s_{n}\right)} \frac{1}{s_{n}^{2}} \sum_{m=0}^{\infty}(-1)^{m}\left(\frac{v s_{n}^{2}}{\lambda}\right)^{m+1} \sum_{k=0}^{m}\left(\begin{array}{l}
m \\
k
\end{array}\right) \lambda_{r}^{k} G_{\alpha, \beta k-m-p+\beta-2, m+1}\left(-\lambda^{-1}, t\right) .
\end{aligned}
$$

The expression of the velocity field can be written as

$$
\begin{aligned}
v(r, t)= & V(r)+\pi \sum_{n=1}^{\infty} \frac{J_{0}^{2}\left(R_{2} s_{n}\right) \phi_{1}\left(s_{n}, r\right)}{J_{1}^{2}\left(R_{1} s_{n}\right)-J_{0}^{2}\left(R_{2} s_{n}\right)}\left[\frac{f_{1}}{\mu s_{n}}\left\{1-\sum_{m=0}^{\infty}(-1)^{m}\left(\frac{v s_{n}^{2}}{\lambda}\right)^{m+1} \sum_{k=0}^{m}\left(\begin{array}{l}
m \\
k
\end{array}\right) \lambda_{r}^{k} G_{\alpha, \beta k-m-2, m+1}\left(-\lambda^{-1}, t\right)\right\}\right. \\
& +\frac{\lambda_{r} f_{1}}{\mu s_{n}} \sum_{m=0}^{\infty}(-1)^{m}\left(\frac{v s_{n}^{2}}{\lambda}\right)^{m+1} \sum_{k=0}^{m}\left(\begin{array}{l}
m \\
k
\end{array}\right) \lambda_{r}^{k} G_{\alpha, \beta k-m+\beta-2, m+1}\left(-\lambda^{-1}, t\right) \\
& -f_{2} \Gamma(p+1) \frac{J_{1}\left(R_{1} s_{n}\right)}{J_{0}\left(R_{2} s_{n}\right)} \sum_{m=0}^{\infty}(-1)^{m}\left(\frac{v s_{n}^{2}}{\lambda}\right)^{m+1} \sum_{k=0}^{m}\left(\begin{array}{l}
m \\
k
\end{array}\right) \lambda_{r}^{k} G_{\alpha, \beta k-m-p-2, m+1}\left(-\lambda^{-1}, t\right) \\
& \left.-\lambda_{r} f_{2} \Gamma(p+1) \frac{J_{1}\left(R_{1} s_{n}\right)}{J_{0}\left(R_{2} s_{n}\right)} \sum_{m=0}^{\infty}(-1)^{m}\left(\frac{v s_{n}^{2}}{\lambda}\right)^{m+1} \sum_{k=0}^{m}\left(\begin{array}{l}
m \\
k
\end{array}\right) \lambda_{r}^{k} G_{\alpha, \beta k-m-p+\beta-2, m+1}\left(-\lambda^{-1}, t\right)\right] .
\end{aligned}
$$

\section{Conclusions and Numerical results}

The purpose of this paper is to establish exact solution for the velocity field corresponding to the flow of Oldroyd-B fluid in the annular region between two infinitely long coaxial cylinders. The motion of the fluid is produced by the inner cylinder pulled with a constant shear and outer cylinder is moving with time dependent velocity. The solution is obtained by Hankel and Laplace transform methods and the result is presented under series form in terms of the generalized-G functions. Plots between various parameters and constants are obtained and relationship has been established.

As shown in below diagrams, the velocity $v(r, t)$ given by Eq. (22) has been drawn against $\mathrm{r}$ for different values of the time $t$, constants and other relevant parameters. The velocity component $v$ is decreasing function of $r$. Figure 1 shows the influence of the time on the fluid motion. As expected, the velocity is increasing function with respect to $t$. The kinematic viscosity $v$, as result from Fig. 2, has a strong influence on the velocity. The result indicates that the velocity is increasing function of $v$. The influences of the relaxation and retardation times on the fluid motion are shown in the figures 3 and 4 . It indicates that the velocity is decreasing function of $\lambda$ and $\lambda_{r}$. Figure 5 show the influence of the fractional parameter $\alpha$ on the fluid motion. It is clearly seen from the figure that the velocity is increasing function of $\alpha$. In figure 6 , it is shown the influence of the fractional parameter $\beta$ on the fluid motion. It is clearly seen from the figure that the velocity is decreasing function $\beta$. Figure 7 show the influences of $p$ on the fluid motion. It is clearly seen from the figure that the velocity is increasing function of $p$. figures 8 and 9show the influences of $f_{1}$ and $f_{2}$ on the fluid motion. Figure 10 show the influence of $\mu$ on the fluid motion. It is clearly seen from the figure that the velocity is increasing function of $\mu$. 


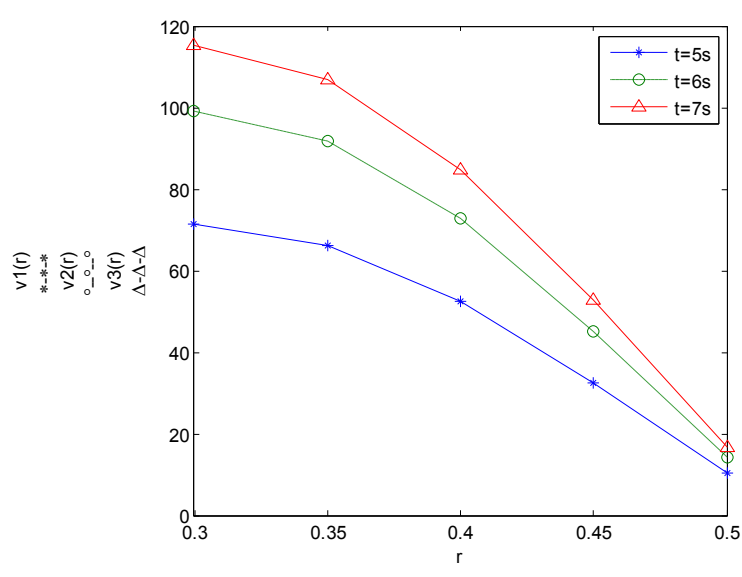

Fig. 1

Fig. 1 Profiles of the velocity v(r, $\mathrm{t})$ given by (22) for $R_{1}=0.3, R_{2}=0.5, f_{1}=3, f_{2}=4, v=0.035$, $\lambda=12, \lambda_{\mathrm{r}}=2.2, \alpha=0.9, \beta=0.6, \mathrm{p}=2, \mu=30$ and different values of $\mathrm{t}$.

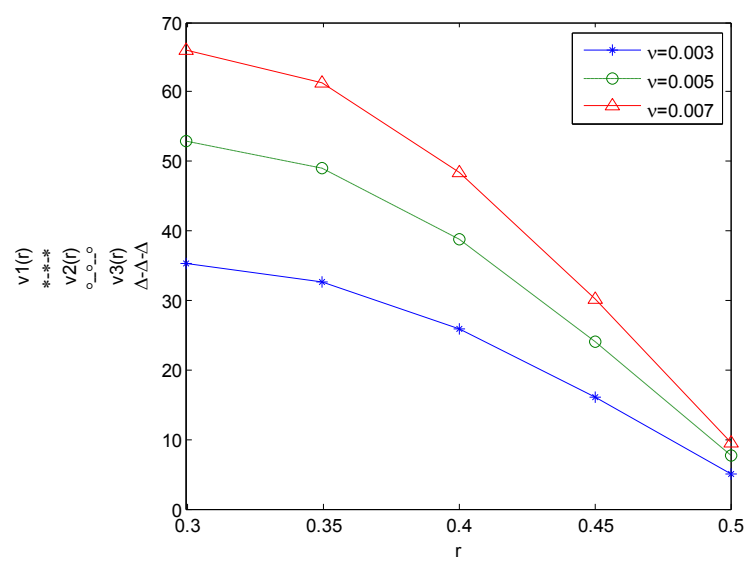

Fig. 2

Fig.2 Profiles of the velocity v(r, t) given by (22) for $R_{1}=0.3, R_{2}=0.5, f_{1}=3, f_{2}=4, \mathrm{t}=6 \mathrm{~s}$, $\lambda=9, \lambda_{\mathrm{r}}=4, \alpha=0.3, \beta=0.3, \mathrm{p}=2, \mu=30$ and different values of $v$.

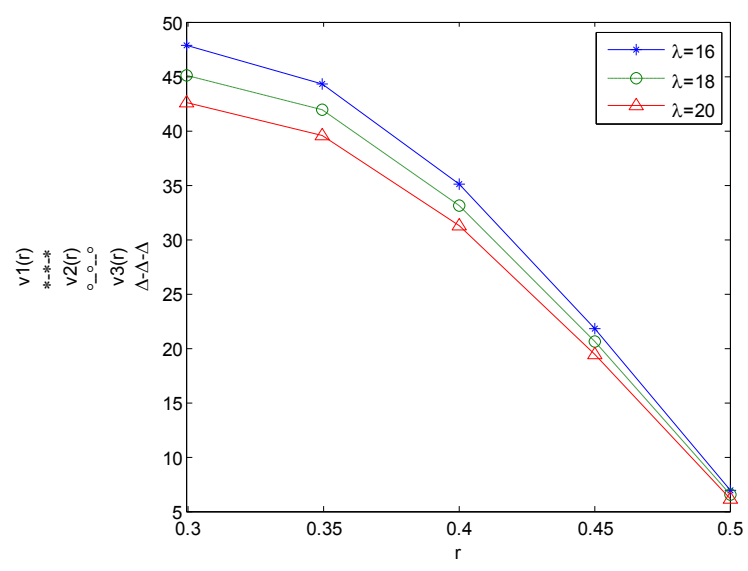

Fig. 3

Fig.3 Profiles of the velocity v(r, t) given by (22) for $R_{1}=0.3, R_{2}=0.5, f_{1}=3, f_{2}=4, \mathrm{t}=5 \mathrm{~s}$, $v=0.01, \lambda_{\mathrm{r}}=7, \alpha=0.3, \beta=0.3, \mathrm{p}=2, \mu=30$ and different values of $\lambda$. 


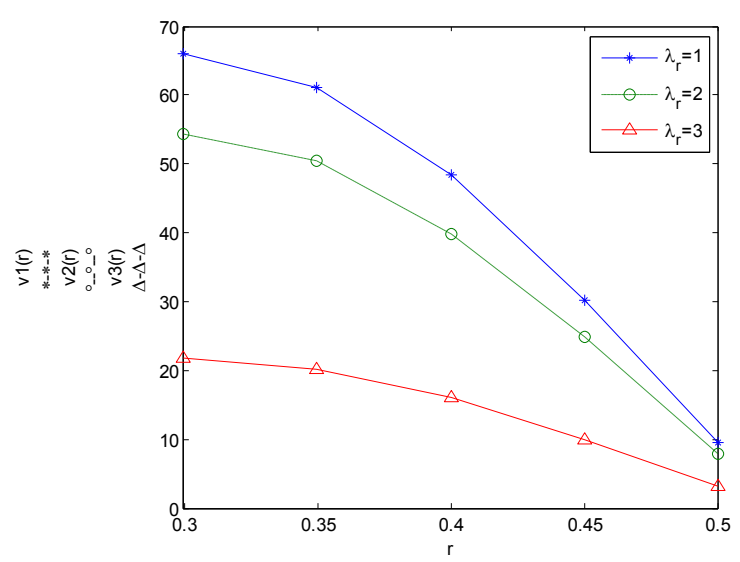

Fig. 4

Fig.4 Profiles of the velocity v(r,t) given by (22) for $R_{1}=0.3, R_{2}=0.5, f_{1}=3, f_{2}=4, \mathrm{t}=5 \mathrm{~s}$, $v=0.04, \lambda=8, \alpha=0.3, \beta=0.9, \mathrm{p}=2, \mu=30$ and different values of $\lambda_{\mathrm{r}}$.

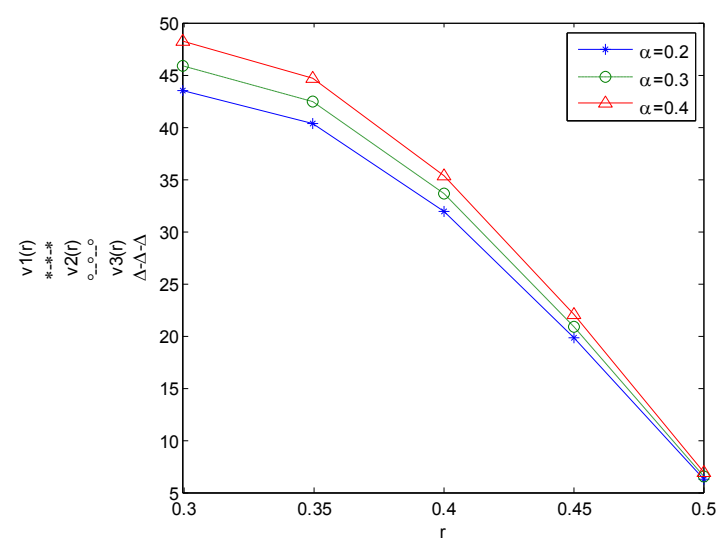

Fig. 5

Fig.5 Profiles of the velocity v(r,t) given by (22) for $R_{1}=0.3, R_{2}=0.5, f_{1}=3, f_{2}=4, \mathrm{t}=6 \mathrm{~s}$, $v=0.01, \lambda=25, \lambda_{\mathrm{r}}=5, \beta=0.5, \mathrm{p}=2, \mu=30$ and different values of $\alpha$.

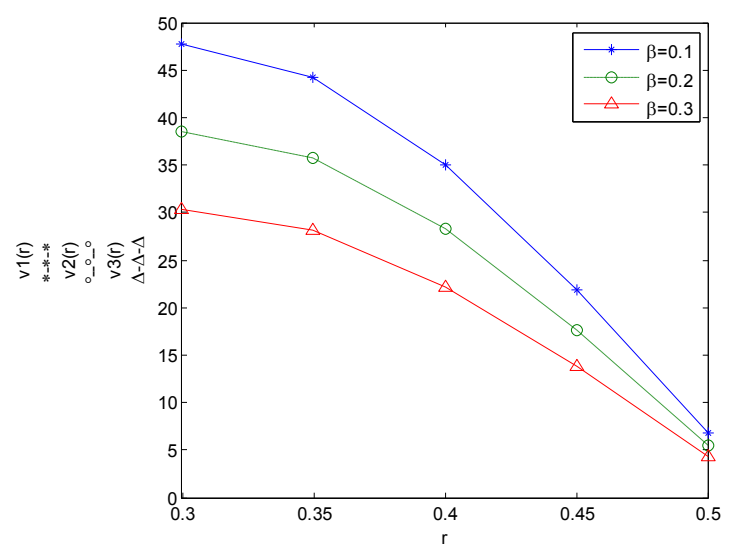

Fig. 6

Fig.6 Profiles of the velocity v(r, t) given by (22) for $R_{1}=0.3, R_{2}=0.5, f_{1}=3, f_{2}=4, \mathrm{t}=6 \mathrm{~s}$, $v=0.04, \lambda=8, \lambda_{\mathrm{r}}=1.5, \alpha=1, \mathrm{p}=2, \mu=30$ and different values of $\beta$. 


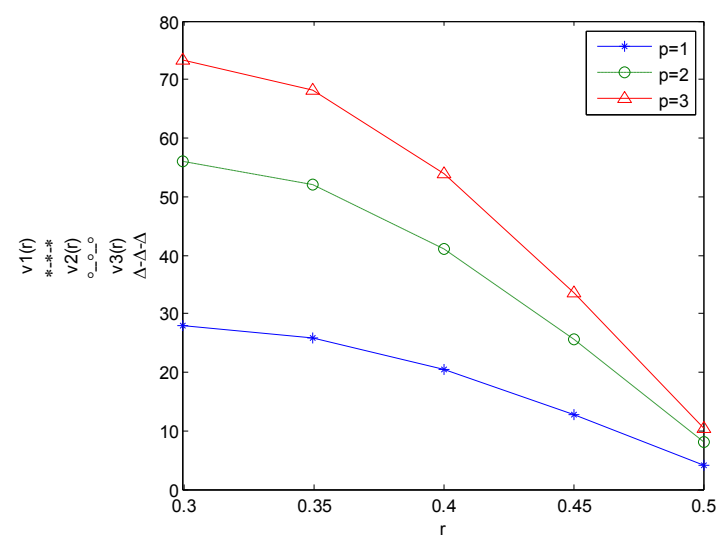

Fig. 7

Fig.7 Profiles of the velocity v(r,t) given by (22) for $R_{1}=0.3, R_{2}=0.5, f_{1}=3, f_{2}=4, \mathrm{t}=5 \mathrm{~s}$, $v=0.04, \lambda=10, \lambda_{\mathrm{r}}=2, \alpha=0.3, \beta=0.9, \mu=30$ and different values of $\mathrm{p}$.

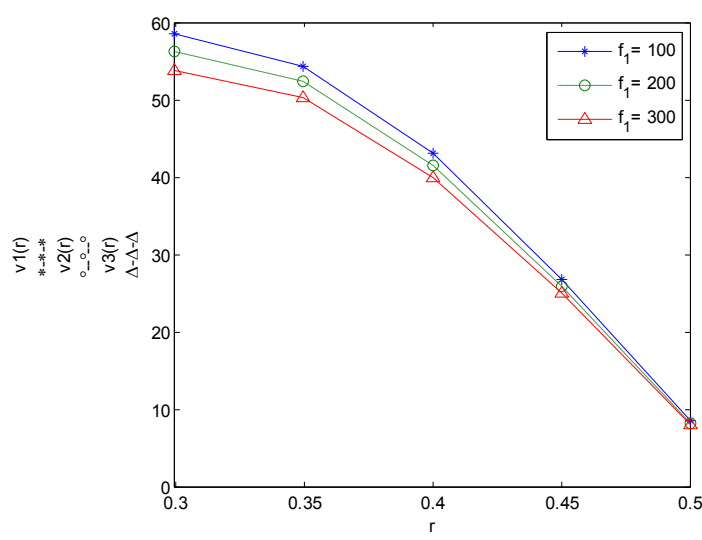

Fig. 8

Fig.8 Profiles of the velocity v(r,t) given by (22) for $R_{1}=0.3, R_{2}=0.5, f_{2}=4, t=5 s, v=0.045$, $\lambda=14, \lambda_{\mathrm{r}}=2.8, \alpha=0.8, \beta=0.5, \mathrm{p}=2, \mu=30$ and different values of $f_{1}$.

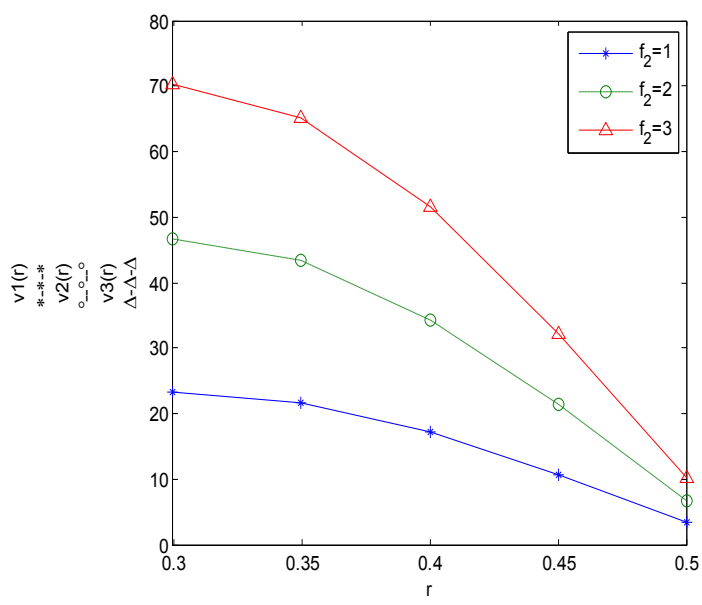

Fig. 9

Fig. 9 Profiles of the velocity $\mathrm{v}(\mathrm{r}, \mathrm{t})$ given by (22) for $R_{1}=0.3, R_{2}=0.5, f_{1}=3, t=6 s, v=0.035$, $\lambda=10, \lambda_{\mathrm{r}}=2, \alpha=0.7, \beta=0.4, \mathrm{p}=2, \mu=30$ and different values of $f_{2}$. 


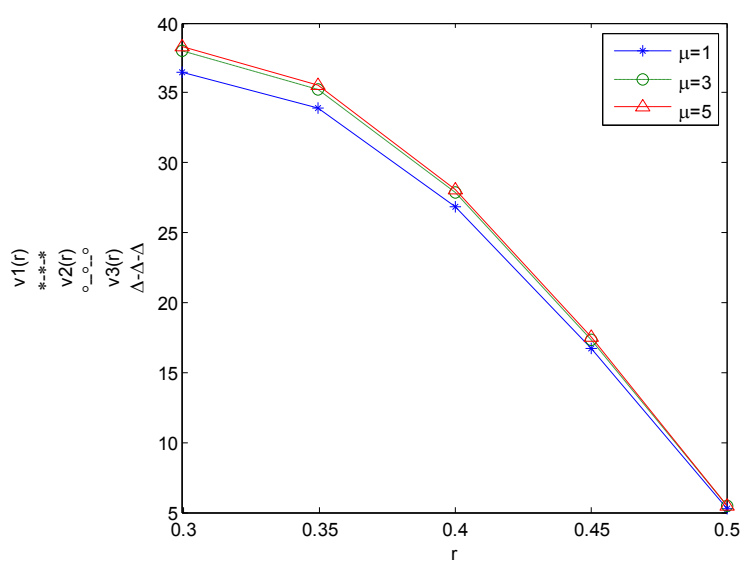

Fig. 10

Fig.10 Profiles of the velocity v(r,t) given by (22) for $R_{1}=0.3, R_{2}=0.5, f_{1}=3, f_{2}=4, t=5 \mathrm{~s}$, $v=0.04, \lambda=11, \lambda_{\mathrm{r}}=2.5, \alpha=0.9, \beta=0.6, \mathrm{p}=2$ and different values of $\mu$.

$$
\text { In all of above, the roots } S_{n} \text { has been approximated by } \frac{(2 n-1) \pi}{2\left(R_{2}-R_{1}\right)}
$$

\section{References}

[1] T.W. Ting, Certain non-steady flows of second-order fluids, Arch. Rational Mech. Anal., 14, 1963, 1-23.

[2] P.N. Srivastava, Non-steady Helical flow of a viscoelastic liquid, Arch. Mech. Stos., 18, 1966, $145-150$.

[3] N.D. Waters and M.J. King, The unsteady flow of an Elastico-viscous liquid in a straight pipe of circular cross section, J. Phys. Appl. Phys., 4, 1971, 204-211.

[4] R. Bandelli and K.R. Rajagopal, Start-up flows of second grade fluids in domains with one finite dimension, Int. J. Non-Linear Mech., 30, 1995, 817-839.

[5] D. Tong, R. Wang and H. Yang, Exact solutions for the flow of non-Newtonian fluid with fractional derivative in an annular pipe, Science in China Ser. G Physics, Mechanics \& Astronomy, 48, 2005, 485-495.

[6] D.K. Tong and Y.S. Liu, Exact solutions for the unsteady rotational flow of non-Newtonian fluid in an annular pipe, Int. J. Eng. Sci., 43, 2005, 281-289.

[7] T. Hayat, S. Nadeem and S. Asghar, Periodic unidirectional flows of a viscoelastic fluid with the fractional Maxwell model, Appl. Math. Compt., 151, 2004, $153-161$.

[8] W.C. Tan and M.Y. Xu, Plane surface suddenly set in motion in a viscoelastic fluid with fractional Maxwell model, Acta Mech. Sinica, 18, 2002, 342-349.

[9] H. Qi and H. Jin, Unsteady rotating flows of a viscoelastic fluid with the fractional Maxwell model between coaxial cylinders, Acta Mechanica Sinica, 22, 2006, 301-305.

[10] M. Kamran, M. Imran, M. Athar and M.A. Imran, On the unsteady rotational flow of fractional Oldroyd-B fluid in cylindrical domains, Meccanica, 47, 2012, 573-584.

[11] M. Amir, C. Fetecau and S. Imran, Exact solutions for some unsteady flows of generalized second grade fluids in cylindrical domains, Journal of Prime Research in Mathematics, 4, 2008, 171-180.

[12] M. Kamran, M. Imran and M. Athar, Exact solutions for the unsteady rotational flow of a generalized second grade fluid through a circular cylinder, Nonlinear Analysis: Modelling and Control, 15, 2010, 437-444.

[13] C. Fetecau, Analytical solutions for non-Newtonian fluid flows in pipe-like domains, Int. J. Non-Linear Mechanics, 39, 2004,225 231.

[14] I. Podlubny, Fractional Differential Equations, Academic Press ( San Diego, 1999).

[15] C.F. Lorenzo and T.T. Hartley, Generalized functions for the fractional calculus, (NASA/TP-1999-209424/REV1, 1999). 